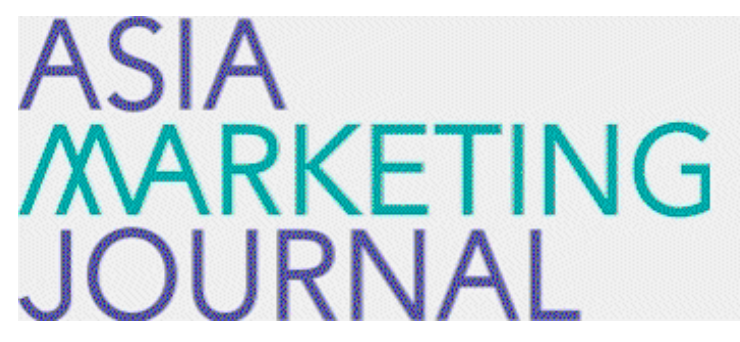

ASIA MARKETING JOURNAL

Volume 17 | Issue 1

Article 4

4-30-2015

\title{
The Lifespan of Social Hub In Social Networking Sites
}

\section{Sangman Han}

Christopher L. Magee

Yunsik Kim

Follow this and additional works at: https://amj.kma.re.kr/journal

Part of the Marketing Commons

\section{Recommended Citation}

Han, Sangman; Magee, Christopher L.; and Kim, Yunsik (2015) "The Lifespan of Social Hub In Social Networking Sites," Asia Marketing Journal: Vol. 17 : Iss. 1 , Article 4.

Available at: https://doi.org/10.15830/amj.2015.17.1.69

This Article is brought to you for free and open access by Asia Marketing Journal. It has been accepted for inclusion in Asia Marketing Journal by an authorized editor of Asia Marketing Journal. 


\title{
The Lifespan of Social Hub In Social Networking Sites: The Role of Reciprocity, Local Dominance and Social Interaction*
}

\author{
Sangman $\mathrm{Han}^{* *}$ \\ Christopher L. Magee ${ }^{* * *}$ \\ Yunsik Kim****
}

\begin{abstract}
This paper examines a highly used social networking site (SNS) by studying the behavior of more than 11 million members over a 20 month period. The importance of the most highly active members to the overall network is demonstrated by the significant fraction of total visits by extremely active members in a given period but such members have surprisingly short lifespans (an average of only 2.5 months) as social hubs. We form and test a number of hypotheses concerning these social hubs and the determinants of their lifespan. We find that the speed of achieving social hub status increases the lifespan of a social hub. The norm of reciprocity is strongly confirmed to be present in the social hub population as visits are reciprocated. We also find that increasing local dominance in terms of activities over neighboring agents leads to a longer lifespan of a social hub. Contrary to expectations, local clustering in the vicinity of social hubs is smaller (rather than larger) than overall clustering. We discuss managerial implications in the paper.
\end{abstract}

Key words: social hubs, social networks, lifespan, norm of reciprocity, dominance

This work was supported by the National Research Foundation of Korea Grant funded by the Korean Government (NRF-2013S1A3A2055050)

** Professor, School of Business, SungKyunKwan University (smhan@skku.edu)

*** Professor, Engineering Systems Division and Director of Center for Innovation in Product Development Massachusetts Institute of Technology, Cambridge (cmagee@mit.edu)

**** Assistant Director, Management Research Institute, School of Business, SungKyunKwan University (yunskim@skku.edu), Corresponding Author 


\section{Introduction}

The rapid recent evolution of SNS (Social Networking Sites) that appear economically viable is particularly noteworthy and such virtual social communities are the principle focus of this work. Such SNS offer personal profiles, blogs, clubs, photos, music, video streams and allow users to submit friends as possibly of interest for their virtual social network. In these sites, young people use digital images, music and postings to express themselves and to share experiences with others. They support personal homepages to strengthen relationships with each other and these homepages enhance their ability to establish an online community. Through maintenance of a personal homepage, users can optimize their self presentation and identity with photos, music and other uploaded information (Katona et al 2011).

Simultaneously with the recognition of the World Wide Web as a social phenomenon, there has arisen a strong use of "Network Analysis" in the study of such large-scale social systems (Watts 2004; Newman 2003). Some of this work has emphasized the existence of power laws in degree distribution (Barabási 2002; Barabási and Bonabeau, 2003; Price 1965, 1976) and have called attention to highly connected nodes in networks (we will call them "social hubs" in this study).

Goldenberg, Han, Lehmann and Hong (2009) explored the role of highly connected hubs in diffusion and adoption. They found that innovative hubs have a greater influence on the speed of the adoption process, and that follower hubs have a greater influence on the size of adoption. Social hubs are likely to play a crucial role in the diffusion of innovation because they occupy a central position in a specific network. Social hubs refer to those actors in a network who make more connections than others in a given time period. These social hubs may be involved in the translation of opinion leadership into a network as opinion leadership is generally correlated with a high average number of network connections (Kratzer and Lettl 2009). Social hubs have been found to play important roles in "epidemic dynamics, innovation diffusion, and synchronization on networks" (Barábasi 2002; Newman 2003) According to Gladwell (2000), social hubs are particularly influential in spreading ideas and behaviors within a social network. Recently, Anagnostopoulos, Kumar and Mahdian (2008) examined social influence between social actors in a social network, interpreting the correlation between the social influence and the social actors' activities in the social system. Iyengar, Van den Bulte and Valente (2010) showed that the amount of social contagion is influenced by the opinion leadership perception of the recipients. Dhanaraj and Parkhe (2006) studied the role of hub firms in innovation networks. They found that "a hub firm can increase the network's dynamic 
stability over time. The hub firm discourages actors' attempts to disconnect ties with the hub firm, and it encourages the formation of new ties, both of which work to grow the network." Hub firms play massive roles in the formation and growth of their networks.

The vitality of a social network is determined by the membership size and the activity of the agents in the network. The most active agents are much more active than the average agent and thus may play a particularly important role in network vitality (Gladwell 2000; Barabási 2002; Kratzer and Lettl, 2009). Our research is based upon the assumption that the lifespan of the most active agents is of particular importance to the long term health of any virtual social network (Barábasi 2002; Dorogovtsev and Mendes 2002, 2003; Newman 2003). We define the lifespan of social hubs as the time interval during which these hubs keep their activity level above a certain threshold level.

In our study of the SNS, we particularly emphasize the lifespan of these highly connected agents over time. Previous studies have found that these highly connected agents are vital in information flow, disease propagation, or wordof-mouth propagation in the network. However, there has been no study on what influences the lifespan of the highly connected agents. Such agents seem to be very important in keeping the network active and appealing, and thus their lifespan has potentially interesting social network and business implications.
Therefore, the focus of this paper is the time dependence of such highly connected agents to examine the nature and determinants of their lifespan. In particular, we explore the influence of three key social network properties - the norm of reciprocity, dominance in the local community, and the local social interaction - on the lifespan of social hubs.

\subsection{Norm of Reciprocity}

Previous studies have found that individuals in real social networks pay close attention to their peers, constantly sending out signals and adjusting their behaviors based on feedback from those with whom they are interacting (Resnick 2004). Positive feedback response from peers is a sign of recognition, approval and even respect for other members of the social network. This feedback response from peers can strengthen the engagement of users and deepen their commitment to the social network. Thus, the more feedback response the users get from their peers, the more time and energy the users spend in the social network, which results in a positive relationship between the level of feedback response and the level of involvement in the community. This phenomenon of feedback and response is called the norm of reciprocity. The norm of reciprocity leads people to match behaviors experienced from others with actions performed for others, giving in proportion to what they receive (Carr 2006). The norm of 
reciprocity has been found in many real communities (Thorn and Connolly 1987; Constant, Sproull, and Kiesler 1996).

It is a general norm that whatever is given ought to be repaid. Onyx and Bullen (2000) found that "individuals operating under a generalized norm of reciprocity provide service to others at a personal cost, but with the expectation that their kindness will be repaid at some point in future." These repayments may be in the form of exchanges in kind or exchanges of some alternate form of aid. In an online peerto-peer file sharing network, Giesler (2006) observed a strong generalized norm of reciprocity, calling it as "an essential stabilizer of that particular social system." Norms of reciprocity predispose individuals to cooperate with each other rather than to treat each other as strangers. Users in the on-line community reciprocate visits to each other, even to those strangers who visit them for the first time. The on-line community is supported by the normative influences that impose a moral responsibility of reciprocity (Giesler 2006; Charla, Wiertz and Ruyter 2008). Constant, Sproull and Kiesler (1996) suggest two explanations for this norm of reciprocity. The first explanation is that the process of reciprocating visits to other members is a means of expressing self identity. By reciprocating the visits to other members, the user can strengthen one's self-identity and attain a certain status. The second explanation is that the norm of reciprocity is a means of showing a strong attachment to the community and a strong bond to the members of the same community.

We hypothesize that agents with hub status are the ones who have a stronger desire to increase self-esteem, gain respect from others and attain status in the social network. This high desire for self-esteem make the social hubs try harder to reciprocate visits they receive from their neighbors. The reciprocity of relationship between the social hubs and their peers is assessed by the balance of incoming visits and outgoing visits of the agents. Thus, we develop the following hypotheses:
H 1a: The number of incoming and outgoing visits for each individual are highly correlated.

H1b: The number of incoming and outgoing visits of social hubs are more balanced than for non-social hubs.

H1c: The more balanced social hubs are in terms of incoming and outgoing visits, the longer lifespan they tend to have.

\subsection{Dominance}

Social hubs, because of their large numbers of connections within the social network, know where the most interesting information is located. Because they enjoy the trust of other members of the network, they are also able to disseminate it to other members more easily (Kleinberg 
1999). This role of social hubs can be strengthened if they dominate their local social network. In other words, the more dominating social hubs are in the relationship within its local network, the more important their role becomes and the more active they become in collecting and disseminating the information. The dominance of social hubs increases as the discrepancy of the activity (incoming visits and outgoing visits) between social hubs and the neighbors in their local social network becomes larger.

The status in a social hierarchy increases selfesteem, and that self esteem results in positive self perceptions of dominance status (Leary, Cottrell and Phillips 2001). People desire dominance status because an individual's status in a dominance hierarchy strengthens his/her perception of self esteem. Thus, motivation through self esteem is ultimately responsible for a need to dominate within the social group. According to Leary, Cottrell and Phillips, "people are motivated to maintain high levels of self esteem because positive self evaluation serves as a subjective monitor of one's relational evaluation - the degree to which other people regard their relationships with the individual to be valuable, important, or close."

Burt (1997) show that value of an individual as an opinion leader is contingent on the number of other people doing the same work. An individual's role in a network becomes more important by bridging structural holes or disconnections between different people in a local network. Individuals with high dominance stand at the crossroads of a large social organization and have the option of bringing disconnections between disconnected people in the network. Thus, the role of social hubs as opinion leaders becomes more important if they dominate their local social network. Stephen and Toubia (2010) also found in on-line commerce that it is better for a shop not to be connected to those shops with high interconnection. In other words, it is best not to be dominated. The shops that benefit most from the network of on-line commerce are those with high number of incoming ties from other shops because the incoming ties increase the accessibility of the shops.

According to Gladwell (2000), the social hubs (described as "connectors" by Gladwell) have mega-influence on their local social network because they are acquainted with an order of magnitude more people than other people. If a social hub is sharing the role of information collection and dissemination with other neighbor members in the same local network, his/ her dominance in the local network will be weakened and he/she becomes less active in information collection and dissemination. This implies that the more an agent dominates their own social network, the more that agent is engaged in the role of collecting and disseminating information in the social network and so longer lifespan is expected. If an existing agent becomes hyperactive much faster, it monopolizes the cumulative advantage mechanism for 
itself. Price (1976) and later Barabási and Albert (1999) argued that when new agents decide where to establish a link, they prefer to attach to an existing agent that already has many other connections. This basic mechanism was called cumulative advantage by Price (1976) and preferential attachment by Barabási and Albert (1999). Thus, if there is only one agent with very high activity in a local social network, the preferential attachment or cumulative advantage will favor only that agent. As a result, the time for this agent to become hyperactive becomes shorter. This social hub, in turn, dominates the local social network, and its role as collector and distributor of information is strengthened. Therefore, the shorter the time to become hyperactive, the more dominating the agents are in their role of collecting and disseminating information, which leads to longer lifespan. The implications of these arguments are contained in the following hypotheses:

H2a: Agents which became hyperactive within a shorter period of time will have longer lifespan as social hubs.

H2b: The more dominant the social hubs are over their local neighbors, the longer will be their lifespan.

\subsection{Local Social Interaction}

Putnam (1995) defined social capital as the features of social organization, such as trust, norms and networks that can improve the efficiency of society by facilitating coordinated actions. Social capital increases as the amount and quality of communication in a community that takes place among its members within the social network (Kavanaugh and Patterson, 2001). The increase in social capital leads to a strong feeling of companionship, emotional bonding, and a sense of belonging, which in turn increases the participation of members in community-related organizations and activities. Coleman (1990) argues that network cohesion should be regarded as the most important source of social capital. Network cohesion is a state in which the people in the network are all interconnected among themselves. According to Coleman, "dense and coherent networks reduce the costs of information searches, promote trust, and facilitate achieving norms." Ellison, Steinfield, and Lampe (2007) examining the relationship between the usage of Facebook and the formation and maintenance of social capital, found a strong association between the usage of SNS and the social interaction. Oh and Jeon (2007) also found that social interaction plays a significant role in sustaining a community. They showed that the dynamic interaction among the members of a community plays a pivotal role in the longevity of the community. Thus, communities with vibrant communication among their members are likely to achieve common social goals and to sustain their longevity. Since the longevity of a community is expected to be correlated with 
the longevity of its central node (Dorogovtsev and Mendes, 2002, 2003), we expect that higher local social interaction leads to longer lifespan of social hubs in the community.

As Putnam (1995) suggested, in order to create social interaction, it is vital to have communities within which members are densely clustered and vibrantly communicate with each other. The clustering coefficients of social hubs indicate the level of connection among their neighbors (Watts and Strogatz, 1998).' Thus, high clustering coefficients of the social hubs imply a higher level of connections within their local networks, which enables vibrant communication within the local network. From these arguments, we develop the following hypothesis:

H3: The higher the clustering coefficients of social hubs, the longer are their lifespan.

\section{Characteristics of the network}

\subsection{Data Set}

The data are obtained from a database $\mathrm{ex}^{-}$ tracted from Cyworld from Dec 2003 to July 2005. Cyworld.com was launched in 1999 and is currently the most popular social network site in Korea with 19 million members in Oct 2006. After formal launch in Korea in 1999, Cyworld was merged in 2004 into nate.com which is a popular portal service in Korea. It has been reported that as much as $90 \%$ of the Korean population in their 20s and a third of the total population of Korea are registered users of Cyworld (CNN 2006). In their homepages, people can accommodate a lot of documents, photos, and appealing items for free but many choose to decorate his/her "minihompy" (Mini homepage) with paid for items. Many people in Korea consider Cyworld as part of everyday life with regard to building relationships with each other and publish his/her daily life on their minihompy to share with others. The number of monthly unique visitors is about 20 million in Cyworld. Cyworld generates revenue from the sale of $\mathrm{cy}^{-}$ ber money which is called Dotori and is worth about 0.3 million dollars a day. The revenue of Cyworld comes from the pay-to-decorate model and the paid advertising model (CNN 2006). Cyworld makes an estimated more than $\$ 7$ per person a year from the pay-to-decorate model.

We obtained anonymous records for 11,163,690 members which is the entire population of cyworld.com for a 20 month period from Dec 2003 to July 2005. ${ }^{2)}$ We studied the network for a series of 1 month periods and define members as agents and a visit to another agent during

1) Clustering coefficient of node $i$ is defined as the ratio of actual to possible links among neighbors of nodes $i$

2) In the data, we excluded 10,074 sites easily identified as nodes with commercial or business purposes by their extreme level of activity and perfect imbalance. 
each 1 month period as a link in our analysis. The link metric for each individual is the number of agents with whom the individual has visited or have been visited for one month period. For example, if person A visits person B twice, person $\mathrm{C}$ once, and person $\mathrm{D}$ three times, and the same person $\mathrm{A}$ is visited by person $\mathrm{B}$ once, by person $C$ twice, and by another person $\mathrm{E}$ twice, then person $\mathrm{A}$ has 3 outgoing links (B, $\mathrm{C}, \mathrm{D})$ and 3 incoming links (B, C, E). Thus, the outgoing links of each person is the number of nodes the person visits during that particular month. The incoming links is the number of people who visit person A during that month. In this paper, we refer to incoming links as Kin, outgoing links as Kout and the total links $K$ is simply Kin plus Kout.

\subsection{Network Characteristics}

Figure 1 is a plot of the degree distribution function $P(x)$, where $x$ is the number of links connecting to given agents. The basic figure is plotted logarithmically on both axes whereas the inset is linear on the $x$ axis. Both representations demonstrate that the distribution has a "wide tail" (more at high $k$ than expected with a normal distribution, Newman 2005). The wide tail is reflected in the fact that some agents are particularly active (more than 1,000

〈Figure 1〉 Degree distribution of total agent population plotted as log-log.

The inset plots the abscissa on a linear scale

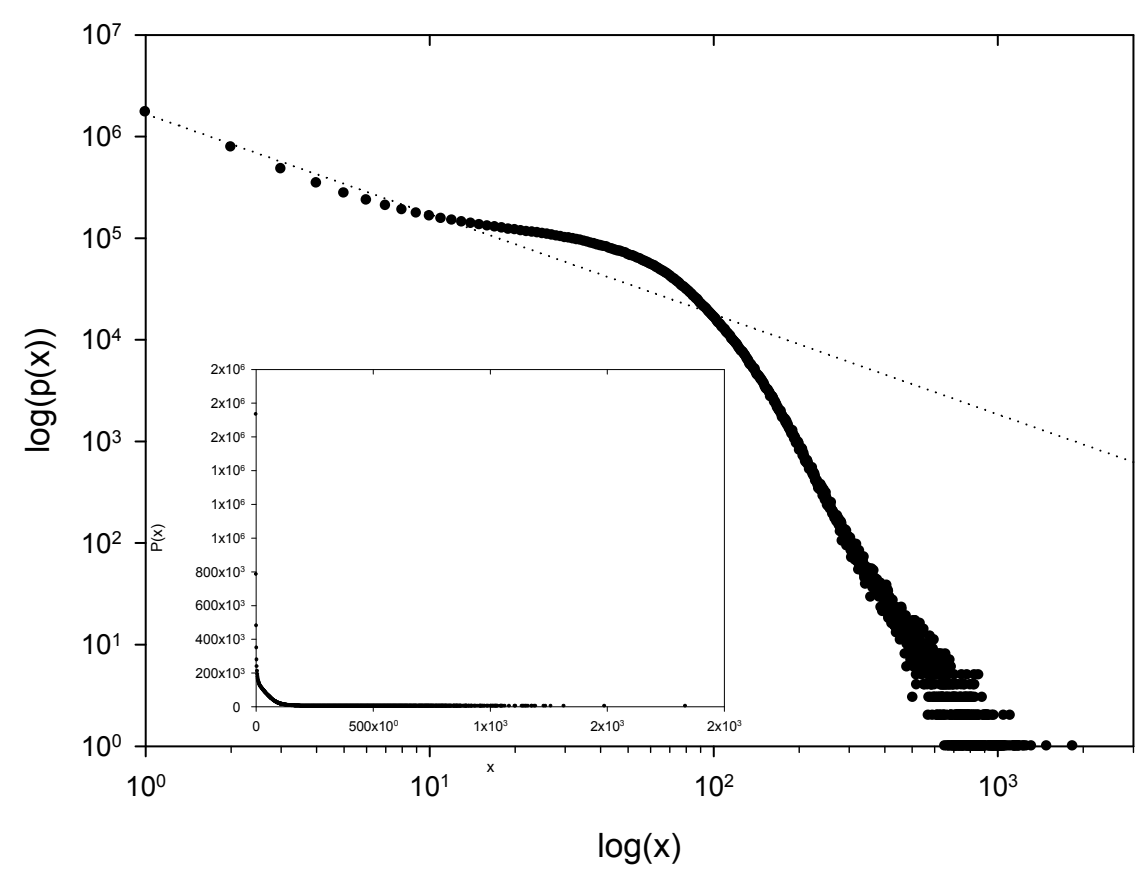

76 ASIA MARKETING JOURNAL Vol. 17 No. 01 April 2015 
visited sites over 20 months) but many other agents are not very active (more than 5 million have less than 10 sites visited over 20 months).

Distributions with particularly wide tails sometimes are described well by power laws. If a power law were followed, the data in Figure 1 would be one straight line on this $\log -\log$ scale as the power law is of form $\mathrm{P}(\mathrm{x}) \approx \mathrm{Cx}^{-\alpha}$, $\ln P(x)=-\alpha \ln x+C$, where $C$ is constant, and $a$ is the power law exponent.

Although our regression showed a reasonable fit $\left(\mathrm{R}^{2}=.91\right)$ with significant parameter values at significant level, a power law clearly only fits for $k$ between around 30 and 800. This is much less than the desired 2 or 3 orders of magnitude range for establishing power law behavior (Newman, 2005). Moreover, the exponent $\alpha$ (3.39) is found to be greater than the range found in other network research (Dorogovtsev and Mendes, 2002, 2003; Barabási et al. 2002; Newman 2003, 2005). The power law exponent of the WWW was found to be $\alpha_{\text {in }}=2.1$ and $\alpha_{\text {out }}=2.45$. Overall, the network we are examining has a wide distribution of activities as for other complex networks (far wider dispersion than a normal distribution) but is not as well-described as a power law as some others are. Since power laws are seen by many as not being a fundamental aspect of system behavior (Doyle et al. 2005), this difference is probably not significant.

\subsection{Social hubs}

We characterize monthly activity in 2 distinct dimensions: incoming links (Kin) and outgoing links (Kout). We classify agents having greater than 1 standard deviation and less than 3 standard deviations above the mean as active and those with higher than 3 standard deviations above the mean as hyperactive. When the three levels of incoming links and another

〈Figure 2〉 Characterization of agents.

Kout

\begin{tabular}{cc}
\hline 3 Std & 1 Std \\
$(57.85)$ & $(27.32)$ \\
\hline
\end{tabular}

\begin{tabular}{|c|c|c|c|c|} 
& & Group 1 & Group 2 & Group 5 \\
\cline { 3 - 5 } Kin & \multirow{3}{*}{$\begin{array}{c}3 \mathrm{Std} \\
(56.64) \\
1 \mathrm{Std} \\
(25.94)\end{array}$} & Group 3 & Group 4 & Group 7 \\
\cline { 2 - 5 } & Group 6 & Group 8 & Group 9 \\
\hline
\end{tabular}

( ) average Kin or Kout over 20 months 
three levels of outgoing links are combined, we have nine categories of agents shown in Figure 2. The measurement of agent status is performed at the level of monthly networks and thus the status of a specific agent varies over time.

Group 1 consists of agents where both Kin and Kout are hyperactive (greater than the mean activity plus 3 standard deviations). Group 2 and 5 refer to agents with hyperactive Kin while group 3 and 6 refer to agents with hyperactive Kout. We define group 1 as balanced social hubs, and groups 2, 3, 5, and 6 as unbalanced social hubs. Figure 3.1 shows the number of balanced social hubs and the number of social hubs over time. Figure 3.2 shows the activities of balanced social hubs and social hubs. The time trends of each class are quite similar and so the number of balanced social hubs to the total membership is nearly constant at about $1.25 \% \pm .15 \%$ over the 20 month time period. However, it is important to note that although the percentage of balanced social hubs remains constant over time, the identities of the social hubs changes from one time to another. This finding is also demonstrated in previous research (Braha and Bar-Yam 2006.)

\section{Methods and Results}

\subsection{Variables and Model Development}

We define lifespan for social hubs as the time

〈Figure 3.1〉 The number of total agents vs. social hubs and balanced social hubs over time.

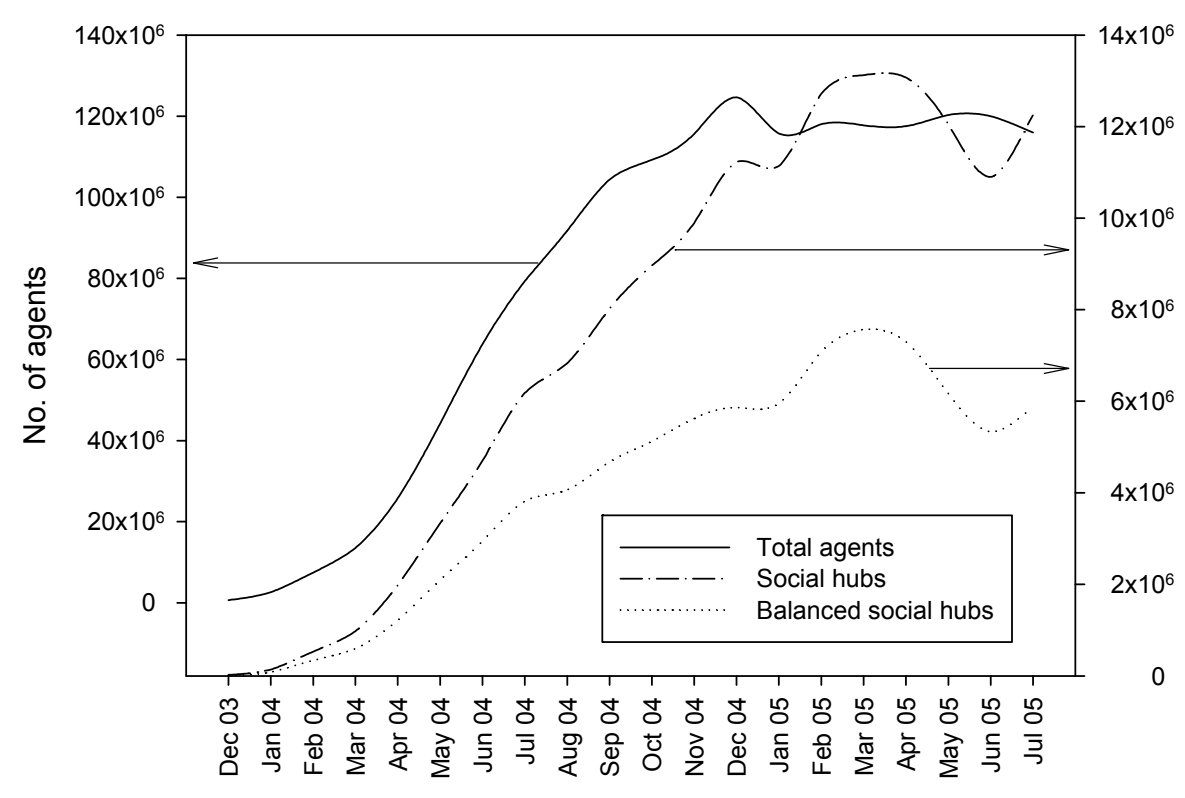

Time 
〈Figure 3.2〉 The activities of total agents vs. social hubs and balanced social hubs over time.

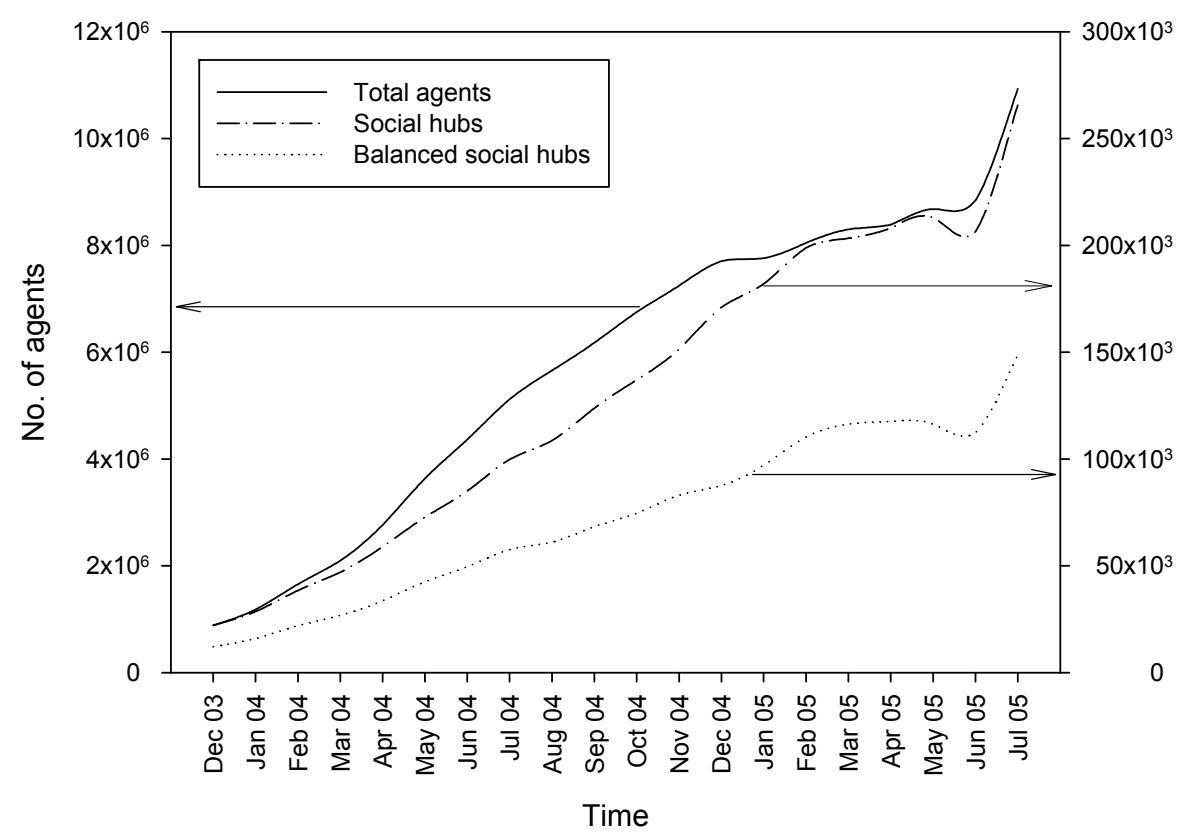

a given agent remains at a social hub status and TBS (Time to Become a Social hub) as the time that was taken to become a social hub for a given agent.

The norm of reciprocity is measured by the extent of imbalance of outgoing visits to incoming visits. Imbalance for the $i$ th agent is defined as the following:

$$
\text { Imbalance }_{\mathrm{i}}=\frac{\sum_{\mathrm{t}=1}^{20} \frac{\left|\operatorname{Kin}_{\mathrm{it}}-\mathrm{Kout}_{\mathrm{it}}\right|}{\operatorname{Kin}_{\mathrm{it}}+\mathrm{Kout}_{\mathrm{it}}}}{\mathrm{T}} \text {, }
$$

where $T$ is the number of months and $i$ is an agent. Imbalance is 1 for agents who only visit others (or who visit no-one but just receives visitors) and is 0 for agents where Kin $=$ Kout.
Dominance is measured by the ratio of the activity of agent $i$ at time $t$ over the average activities of its neighbor agents at time $t$. Dominance is defined as the following:

$$
\text { Dominance }_{\mathrm{it}}=\frac{\text { Activity }_{\mathrm{it}}}{\text { Act Neigh }_{\mathrm{it}}} \text {, }
$$

where Activity $_{\text {it }}$ is the activity of agent $i$ at time $t$ and Act Neigh $h_{i t}$ is the average activities of neighbor agents at time $t$. Local social interaction is an element of social capital and in this work is measured by local clustering coefficients introduced by Watts and Strogatz (1998). Following Watts and Strogatz, we define the clustering coefficient for agent $i$ in terms of the interconnection among agent is 
neighbors. If $k_{i t}$ is the number of neighbor agents of agent $i$ at time $t, k_{i t}\left(k_{i t}-1\right) / 2$ links can exist among these neighbors. Let $n_{i t}$ be the actual number of links among the neighbor agents at time $t$. Then, the clustering coefficient can be measured as the ratio of actual to possible links among neighbors of agents $i$ at time t. Clustering coefficient is defined as the following:

$$
\mathrm{CC}_{\mathrm{it}}=\frac{\mathrm{n}_{\mathrm{it}}}{\mathrm{k}_{\mathrm{it}}\left(\mathrm{k}_{\mathrm{it}}-1\right) / 2} \text {, }
$$

If every neighbor connected to node $i$ is also connected to all other neighbors of node $i$, then $\mathrm{CC}$ becomes 1 . If none of the nodes connected to node $i$ is connected to each other, then CC becomes 0 .

Table 1 gives a summary of the variables. As the variables such as imbalance, dominance and $\mathrm{CC}$ are time dependent, we have to consider the group that specific agents belong to at the various times. For calculation over the entire time period we take the weighted average to calculate the average of the variables when some agents belong to more than 2 groups over time. If all the weights are equal, it is the same as an arithmetic mean. This is accomplished by defining the weight $W_{i j}$ as the proportion of each group $j$ in the lifespan of agent $I$.

$$
\mathrm{w}_{\mathrm{ij}}=\frac{\text { Lifespan }_{\mathrm{ij}}}{\sum_{\mathrm{j}=1}^{\mathrm{n}} \operatorname{Lifespan}_{\mathrm{ij}}}
$$

The variables in Table 1 are defined by the following equations.

$$
\begin{aligned}
& \text { Imbalance }_{\mathrm{i}}=\sum_{\mathrm{j}=1}^{\mathrm{n}} \mathrm{w}_{\mathrm{ij}} \text { Imbalance }_{\mathrm{ij}} \\
& \text { Dominance }_{\mathrm{i}}=\sum_{\mathrm{j}=1}^{\mathrm{n}} \mathrm{w}_{\mathrm{ij}} \frac{\text { Activity }_{\mathrm{ij}}}{\text { Act Neigh }_{\mathrm{ij}}},
\end{aligned}
$$

where Activity $_{i j}$ is the activity of agent $i$, and Act $N e i g h_{i j}$ is the average activities of neighbor agents of agent $i$ when it belongs to group $j$. The groups are defined based on the size of incoming visits (Kin) and outgoing visits (Kout). A social hub may belong to group $1,2,3$, 5, or 6. Group 1 is the balanced social hub group, and groups 2, 3, 5 and 6 are the unbalanced social hub groups.

$$
\mathrm{CC}_{\mathrm{i}}=\sum_{\mathrm{j}=1}^{\mathrm{n}} \mathrm{w}_{\mathrm{ij}} \mathrm{CC}_{\mathrm{ij}} \text {, }
$$

where $j$ stands for groups, and $i$ stands for an agents.

We used a survival analysis for studying the lifespan of social hubs. The lifespan is right censored because the status of a social hub is terminated at the end of the data period. We used the proportional hazard model, which assumes a parametric form for the effects of the predictors on the hazard function and make no assumption about the form of the survival function $(\mathrm{h}(\mathrm{t}))$ (Cox 1972). It is a common method of representing probability distribution of dura- 
〈Table 1〉 Summary of variables on analysis

\begin{tabular}{cc}
\hline Variable & Variable description \\
\hline Lifespan $_{i}$ & Time to remain a social hub status for agent $i$. \\
TBS $_{i}$ & Time to become a social hub for agent $i$. \\
Dominance $_{i}$ & The ratio of agent $i$ s activities to agent is neighbors activities \\
Imbalance $_{i}$ & Extent of balance of outgoing visits to incoming visits, 0 means very balanced \\
$\mathrm{CC}_{i}$ & and 1 very unbalanced. \\
Gender & Clustering coefficient for agent $i$. \\
Age & Categorical variable (male 0, female 1$).$ \\
\hline
\end{tabular}

tion times and it works well with handling of censoring (Allison 1995; Reinartz and Kumar 2003). The hazard rate for agent $i$ is assumed to take the following form:

$$
\begin{aligned}
\mathrm{h}_{\mathrm{i}}(\mathrm{t})= & \mathrm{h}_{0}(\mathrm{t}) \exp \left(\beta \chi_{\mathrm{i}}\right) \\
\mathrm{h}_{\mathrm{i}}(\mathrm{t})= & \mathrm{h}_{0} \exp \left(\beta_{1} \text { TBS }_{\mathrm{i}}+\beta_{2} \text { Dominance }_{\mathrm{i}}\right. \\
& +\beta_{3} \text { Imbalance }_{\mathrm{i}}+\beta_{4} \mathrm{CC}_{\mathrm{i}}+\beta_{5} \text { Gender }_{\mathrm{i}} \\
& \left.+\beta_{6} \text { Agel }_{\mathrm{i}}+\beta_{7} \text { Age }_{\mathrm{i}}+\beta_{8} \text { Age }_{\mathrm{i}}\right)
\end{aligned}
$$

$h_{0}(t)$ is the baseline hazard function which indicates the effect of independent variables on hazard rate and $\beta X_{i}$ is the impact of explanatory variables. We estimated the proportional hazard model using the partial likelihoo11d method. We handled ties using the Efron method instead of the Breslow approximation because data for the lifespan of social hubs and balanced social hubs are heavily tied (Farewell and Prentice 1980; Allison 1995).

\subsection{Properties of Social hubs}

The data are obtained from a database extracted from Cyworld from Dec 2003 to July 2005 for 20 months and the total number of agents used for our analysis is 11,163,690. The number of social hubs is 468,278; among them 230,491 (49.22\%) are balanced social hubs. Table 2 describes the demographic properties of social hubs, balanced social hubs and the overall population. Members in the 18-24 age group are more strongly represented in the social hub group than in the overall population of the social network. In the overall population, the proportion of $18-24$ age group is $31.87 \%$, while the same age group represents $67.56 \%$ in the social hub group. This figure becomes even higher for balanced social hubs (71.55\%). A Chi-square test for the difference of the 18-24 age group participation between social hubs and overall population shows that there is a significant difference $\left(x^{2}=257,717, p<.001\right)$ between the two groups. Mikami (2002) showed that people 
of age 18-24 spend the most time socializing with friends in both the U.S. and Japan. Since those in late teens and early twenties are also voracious users of new technologies - in particular, Internet related technologies such as SMS, Instant message service, on-line community, etc, they are more frequent and intense users of on-line social networks. Both of the two effects described above-1) more propensities for socializing with friends and 2) more frequent and intense use of Internet-related technologies make those in their late teens and early twenties likely to have higher activity levels in specific on-line social network sites- a conclusion corroborated by our results

Table 2 also shows that the composition of females is slightly larger than that of males (51.5\% for female and $48.5 \%$ for male) in social hubs. For the entire population, the proportion of $\mathrm{fe}^{-}$ male is slightly smaller than that of male $(49.9 \%$ for female and $50.1 \%$ for male).

Table 3 and Figure 4.1 and 4.2 summarize the characteristics of social hubs. Table 3 shows that social hubs do not have extensive lifespan. It takes an average of about 7.14 months after they become a member until they reach social hub status. On average, they stay for only 2.45 months as social hubs. The average activity level (sum of Kin and Kout) is 122 for social hubs and 141 for balanced social hubs. Social hubs are in general balanced (the imbalance score is only 0.15 ). Figure 4.1 and 4.2 show the distribution of lifespan and TBS (Time to Become a Social hub) of social hubs. It shows that most social hubs have short lifespans (less than or equal to 2 months) while a few have a long lifespan of more than 12 months. It also shows that the time to become social hubs ranges between 1 month and 20 months with a mode of 5 months (7 months on average).

The lifespan of social hubs is far shorter than we expected. One of the reasons could be the extreme definition of social hubs. We define social hubs as those whose Kin or Kout is at

〈Table 2〉 Demographics of agents

\begin{tabular}{cccc}
\hline & Social hubs & Balanced social hubs & Total agents \\
\hline Number of agents & $468,278(4.19 \%)$ & $230,491(2.06 \%)$ & $11,163,690$ \\
Age & 22.79 & 22.69 & 25.74 \\
13 to 17 & $3.38 \%$ & $2.06 \%$ & $10.76 \%$ \\
18 to 24 & $67.56 \%$ & $71.55 \%$ & $31.87 \%$ \\
25 to 29 & $25.13 \%$ & $23.37 \%$ & $28.82 \%$ \\
30 to 39 & $3.93 \%$ & $3.02 \%$ & $28.56 \%$ \\
Sex & & & \\
Male & $48.49 \%$ & $49.01 \%$ & $50.11 \%$ \\
Female & $51.51 \%$ & $50.99 \%$ & $49.89 \%$ \\
\hline
\end{tabular}


least 3 standard deviation away from its mean, and we measure the lifespan during which they retain the status of social hubs. A second reason could be the ceiling effects of the reciprocal behaviors of social hubs. Ceiling effects imply that there exists some kind of an upper limit of time and efforts for social hubs to spend in order to maintain the norm of reciprocity. Another reason could be the tendency of regressing to the mean activity level for the users. The results suggest that social hubs have diffi- culty maintaining their highly active and reciprocal behavior for more than 2.5 months on average.

\subsection{Test of the Norm of Reciprocity Assumption}

In hypothesis 1a, the incoming and outgoing visits are expected to be highly correlated (Barabási 2002: Newman 2003; Braha and Bar-Yam 2006). This hypothesis is based on

〈Table 3〉 Descriptive analysis of social hubs and balanced social hubs

\begin{tabular}{ccccc}
\hline & \multicolumn{2}{c}{ Social hubs } & \multicolumn{2}{c}{ Balanced social hubs } \\
\hline Variable & Mean & SD & Mean & SD \\
Lifespan & 2.4546 & 2.0994 & 2.3932 & 2.0014 \\
TBS & 7.1473 & 4.2889 & 7.0309 & 4.2684 \\
Dominance & 1.8527 & .8828 & 1.9946 & .6602 \\
Imbalance & .1514 & .2005 & .0669 & .0639 \\
CC & .0783 & .0494 & .0799 & .0430 \\
\hline
\end{tabular}

〈Figure 4.1〉 Lifespan distribution for social hubs

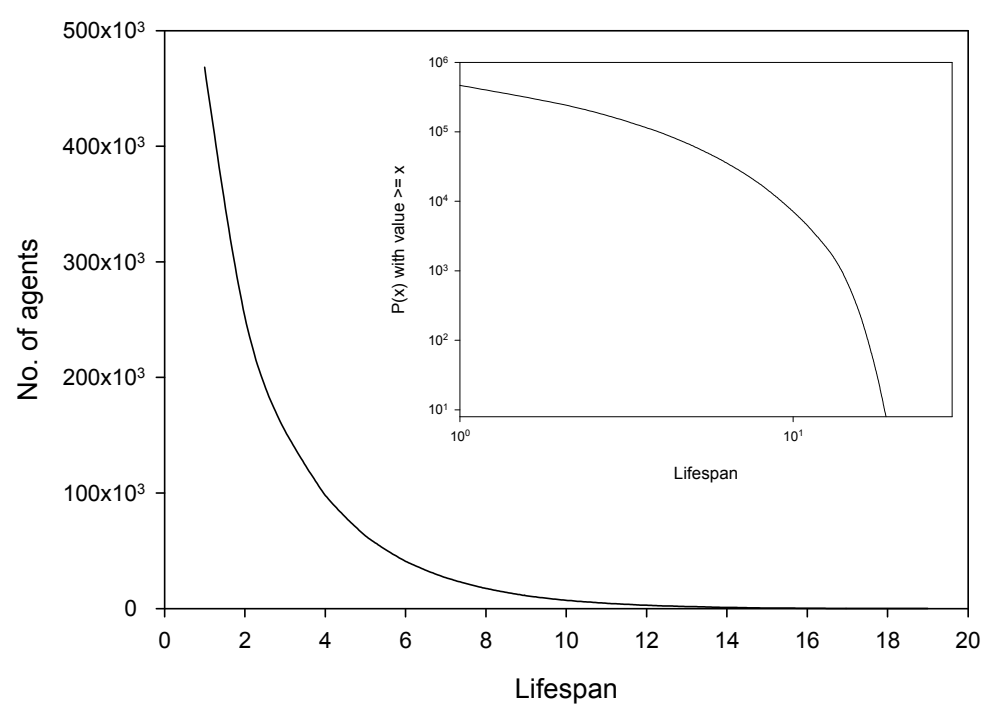

The Lifespan of Social Hub In Social Networking Sites: The Role of Reciprocity, Local Dominance and Social Interaction 83 
〈Figure 4.2〉 TBS distribution for social hubs

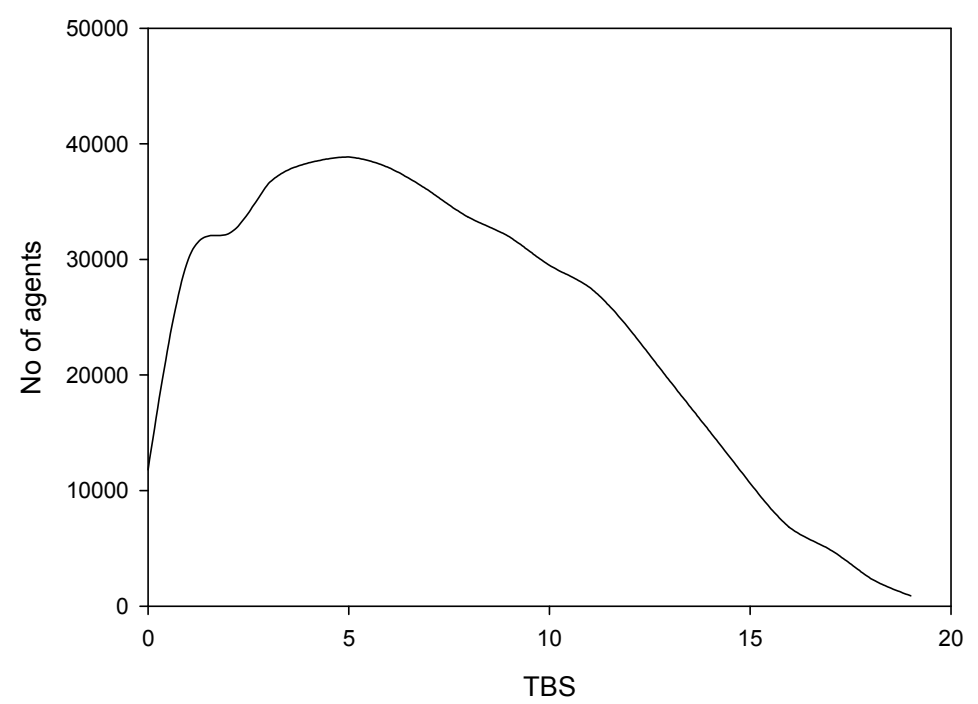

the notion of the norm of reciprocity. The norm of reciprocity is regarded as a general principle of behavior both in a real community and in a virtual social network (Wellman and Gulia, 1999; Constant, Sproull, and Kiesler, 1996). In order to test whether this is true in our dataset, we run a simple regression of Kin on Kout. If the general principle holds in the virtual social network we study, we expect the coefficient of Kout to be close to 1 . We run this simple regression on all 11,163,690 members. The regression is as follows:

$$
\text { Kin }_{i t}=\alpha_{\mathrm{t}}+\beta_{\mathrm{t}} \text { Kout }_{i t}
$$

$K_{i n}$ (and Kout $t_{i t}$ ) in this equation means the number of other agents who visited the agent $i$ in period $t$ (and number of other agents whom agent $i$ visited in period $t$ ). The regression is for all agents over the entire 20 month period. "Perfect Reciprocity" should yield $\alpha_{\mathrm{t}}=0, \beta_{\mathrm{t}}$ $=1$ and $\mathrm{R}^{2}=1$; "Perfect Non-reciprocity" should yield $\alpha_{\mathrm{t}}=$ average of Kin, $\beta_{\mathrm{t}}=0$ and $\mathrm{R}^{2}=0$.

In table 4, we list the coefficients of Kout, $\mathrm{R}^{2}$, and average of Kin and Kout over 20 months. For the entire period, $\alpha$ is 1.13 (vs. 8.17 for average of Kin), $\beta$ is .853 and $\mathrm{R}^{2}$ is .853 showing very strong support for the norm of reciprocity in the network. The monthly results similarly show that the norm of reciprocity holds in shorter time periods as well. The coefficients are in the range of $.73-.88$, and $R^{2}$ is also quite high (.71-.88). Thus, the long-known "norm of reciprocity" is found to be very well followed in this social network.

Next, we tested hypothesis $1 \mathrm{~b}$ to see whether social hubs are more balanced than non-social 
$\langle$ Table 4〉 Regression analysis of Kin on Kout for all agents

\begin{tabular}{ccccccc}
\hline Period (t) & $\alpha_{\mathrm{t}}$ & $\beta_{\mathrm{t}}$ & $\mathrm{R}^{2}$ & $\begin{array}{c}\text { Average of } \\
\text { Kin }\end{array}$ & $\begin{array}{c}\text { Average of } \\
\text { Kout }\end{array}$ & $\begin{array}{c}\text { Correlation } \\
\text { between Kin } \\
\text { and Kout }\end{array}$ \\
\hline Month1 & .4727 & .7972 & .8165 & 3.0107 & 3.1834 & .904 \\
Month2 & .6100 & .8375 & .8648 & 4.5050 & 4.6507 & .930 \\
Month3 & .7097 & .8858 & .8833 & 6.2491 & 6.2532 & .940 \\
Month4 & .9067 & .8711 & .8687 & 7.4498 & 7.5114 & .932 \\
Month5 & 1.1826 & .8573 & .8371 & 9.1508 & 9.2949 & .915 \\
Month6 & 1.4881 & .8535 & .8367 & 10.7635 & 10.8670 & .915 \\
Month7 & 1.8319 & .8394 & .8267 & 11.9765 & 12.0862 & .909 \\
Month8 & 2.1528 & .8201 & .8112 & 12.0996 & 12.1291 & .901 \\
Month9 & 2.1525 & .8190 & .8087 & 12.1917 & 12.2579 & .899 \\
Month10 & 2.3995 & .8059 & .7965 & 12.4087 & 12.4197 & .892 \\
Month11 & 2.4357 & .7967 & .7860 & 11.7430 & 11.6823 & .887 \\
Month12 & 2.4879 & .7898 & .7748 & 11.4830 & 11.3893 & .880 \\
Month13 & 3.0301 & .7475 & .7382 & 11.6520 & 11.5340 & .859 \\
Month14 & 2.7658 & .7466 & .7291 & 10.5854 & 10.4731 & .854 \\
Month15 & 2.5907 & .7596 & .7428 & 10.3687 & 10.2400 & .862 \\
Month16 & 2.3361 & .7795 & .7525 & 10.0429 & 9.8866 & .867 \\
Month17 & 2.5627 & .7507 & .7287 & 9.8469 & 9.7029 & .854 \\
Month18 & 2.6632 & .7411 & .7174 & 9.7864 & 9.6118 & .847 \\
Month19 & 2.7053 & .7320 & .7108 & 9.5929 & 9.4097 & .843 \\
Month20 & 2.4218 & .7401 & .7208 & 8.0170 & 7.5596 & .849 \\
Entire Period & 1.1261 & .8528 & .8526 & 8.1748 & 8.1073 & .887 \\
\hline
\end{tabular}

hubs in terms of incoming and outgoing visits. Table 5 contains average imbalance, actual and expected number (and proportion) of members over 20 months for each group of social hubs.

The "expected" number in this table is that for a normal distribution. Groups 1, 2, and 3those groups with low scores of imbalance (which means they are more balanced) show the largest discrepancies between actual and expected proportion of members. In particular, the expected proportion of social hubs in groups 1,2 and 3 is about 5 orders of magnitude greater than that expected randomly. Some of this discrepancy is due to the wide tail in the degree distribution shown in Figure 1 but not all of it. For example, groups 5 and 6 show large scores of imbalance (.5726 and .8456).and their actual proportion is only one order of magnitude different from the expected random proportion. This results show that social hubs are far more abundant than would be expected, and consequently confirm that the norm of reciprocity 
prevails in the group of social hubs. We can also compare the imbalance score of social hubs with that of other agents. The imbalance score of social hubs (group1, 2, 3, 5, and 6, imbalance $=.15$ ) turned out to be much lower than the other agents (group 4, 7, 8, and 9, imbalance $=.53$ ), which supports our hypothesis that social hubs try harder to reciprocate the visits from other agents. Moreover, we find overall that in any given month, there is $40 \%$ probability of a specific agent returning a visit to another specific agent, in other words, about $40 \%$ of visits between specific agents is reciprocated in any given month.

\subsection{Factors influencing Lifespan of Social hubs}

In this section, we perform a proportional hazard model analysis on 468,278 social hubs and on 230,491 balanced social hubs respectively. Table 6 shows the correlation analysis of the variables we used in the proportional hazard model. It is interesting that the network- re- $^{-}$ lated behaviors of those in the age range 18-24 are strikingly different from those in the age range 25-29. Imbalance, dominance and clustering coefficients among social hubs as well as balanced social hubs are significantly correlated with opposite signs between the two age groups (age 18-24 and age 25-29). Those in 18-24 tend to have lower imbalance (higher norms of reciprocity), higher clustering coefficients (more densely connected local network), and lower dominance over their local neighbors, while those in the age of 25-29 tend to have higher imbalance (lower norms of reciprocity), lower clustering coefficients (less densely connected local network) and higher dominance over their local neighbors. These results indicate that norm of reciprocity and general social interaction is more important for those in their late teen and early twenties, while dominance appears more important for those in their late twenties as their motivation to become social hubs.

We report the results of the lifespan model in Table 7. It is important to note that signs of coefficients have been reversed to reflect the effects on lifespan. We calculated explanatory power $(|\beta| \mathrm{S})$ which is the relative importance of variables in explaining variation on agent's lifespan, where $\mathrm{S}$ is the standard deviation for

〈Table 5〉 The imbalance, and actual vs. expected number of membership for each social hub group

\begin{tabular}{cccccc}
\hline & Group1 & Group2 & Group3 & Group5 & Group6 \\
\hline Imbalance & .0669 & .0683 & .1744 & .5726 & .8456 \\
\hline Actual Number & 230,491 & 165,762 & 196,332 & 8,368 & 30,113 \\
and proportion & $1.56 \%$ & $1.12 \%$ & $1.33 \%$ & $.06 \%$ & $.20 \%$ \\
\hline Expected Number & 0.5 & 2.0 & 2.3 & 909.3 & 1603.8 \\
and proportion & $.00001 \%$ & $.00002 \%$ & $.00002 \%$ & $.00814 \%$ & $.01437 \%$ \\
\hline
\end{tabular}


〈Table 6〉 Correlation analysis between variables

a. Balanced social hubs

\begin{tabular}{|c|c|c|c|c|c|c|c|c|}
\hline Lifespan & & & & & & & & \\
\hline TBS & -.26 & & & & & & & \\
\hline Dominance & .10 & -.06 & & & & & & \\
\hline Imbalance & -.02 & .01 & .58 & & & & & \\
\hline $\mathrm{CC}$ & -.12 & -.02 & -.47 & -.35 & & & & \\
\hline Gender & .03 & .05 & -.03 & -.11 & -.03 & & & \\
\hline agel(13-17) & -.07 & .01 & .01 & .05 & .10 & .08 & & \\
\hline age2(18-24) & .03 & .12 & -.4 & -.18 & .26 & .10 & -.23 & \\
\hline age3(25-29) & 0 & -.11 & .34 & .14 & -.28 & -.13 & -.08 & -.88 \\
\hline
\end{tabular}

b. Social hubs

\begin{tabular}{|c|c|c|c|c|c|c|c|c|}
\hline Lifespan & & & & & & & & \\
\hline TBS & -.29 & & & & & & & \\
\hline Dominance & .05 & -.02 & & & & & & \\
\hline Imbalance & -.08 & -.01 & .08 & & & & & \\
\hline CC & -.06 & -.02 & -.29 & -.27 & & & & \\
\hline Gender & .02 & .05 & -.02 & -.05 & .01 & & & \\
\hline age1(13-17) & -.09 & 0 & 0 & .03 & .09 & .10 & & \\
\hline age2(18-24) & .03 & .09 & -.29 & -.15 & .24 & .10 & -.27 & \\
\hline age3(25-29) & .02 & -.08 & .23 & .10 & -.25 & -.15 & -.11 & -.84 \\
\hline
\end{tabular}

each variable (Bolton 1998). Table 7 shows that

both imbalance (norm of reciprocity) and dominance are highly significant and consistent with our hypothesis. However, the clustering $\mathrm{co}^{-}$ efficient (local social interaction) is significant, but has a negative coefficient. This means that local social interaction has negative influence on the lifespan of social hubs. The relative influence on the lifespan of social hubs is in the order of age dummy of 18-24 (24.02\%), TBS (19.04\%) and imbalance (13.67\%), followed by Clustering coefficient (12.17\%) and dominance
$(8.68 \%)$.

Both dominance and imbalance are significant and consistent with the hypotheses $(\beta=.072$, $p<.01$ for dominance, $\beta=-.512, p<.01$ for imbalance). TBS is also significant and consistent with the relevant hypothesis $(\beta=-.032$, $p<.01)$. Thus, the faster an agent becomes a social hub, and the more dominant a social hub is over its local neighbors, s/he tends to stay longer as a social hub. Hypotheses are also supported for the balanced social hubs. TBS, dominance, and imbalance are significant and 
$\langle$ Table 7〉 Cox Proportional Hazard Model Estimation Results for the lifespan of social hubs

\begin{tabular}{|c|c|c|c|c|c|c|}
\hline & \multicolumn{3}{|c|}{ Social hubs } & \multicolumn{3}{|c|}{ Balanced social hubs } \\
\hline Variables & Coefficients ${ }^{\dagger}$ & $\begin{array}{l}\text { Standard } \\
\text { Error }\end{array}$ & $\begin{array}{c}\text { Explanatory } \\
\text { Power }\end{array}$ & Coefficients ${ }^{\dagger}$ & $\begin{array}{l}\text { Standard } \\
\text { Error }\end{array}$ & $\begin{array}{c}\text { Explanatory } \\
\text { Power }\end{array}$ \\
\hline TBS & $-.032 * *$ & .000 & $18.49 \%$ & $-.028 * *$ & .001 & $12.04 \%$ \\
\hline Dominance & $.072^{* *}$ & .003 & $8.39 \%$ & $.253^{* *}$ & .006 & $16.79 \%$ \\
\hline Imbalance & $-.512^{* *}$ & .008 & $13.63 \%$ & $-1.906^{* *}$ & .054 & $11.50 \%$ \\
\hline $\mathrm{CC}$ & $-1.870^{* *}$ & .040 & $12.44 \%$ & $-3.408^{* *}$ & .067 & $13.71 \%$ \\
\hline $\begin{array}{c}\text { Gender } \\
\text { Age }\end{array}$ & $.065^{* *}$ & .003 & $4.30 \%$ & $.023^{* *}$ & .005 & $1.15 \%$ \\
\hline $13-17$ & $.434^{* *}$ & .014 & $10.39 \%$ & $.997 * *$ & .027 & $14.04 \%$ \\
\hline $18-24$ & $.385^{* *}$ & .009 & $24.11 \%$ & $.549 * *$ & .014 & $24.83 \%$ \\
\hline $25-29$ & $.144^{* *}$ & .009 & $8.25 \%$ & $.141^{* *}$ & .014 & $5.94 \%$ \\
\hline$-2 L L$ & $8,727,771.20$ & & & $4,226,220.50$ & & \\
\hline
\end{tabular}

consistent with the hypotheses $(\beta=-.028, p<$ .01 for TBS, $\beta=.253, p<.01$ for dominance, $\beta=-1.906, p<.01$ for imbalance). Both dominance and imbalance (norm of reciprocity) have larger coefficients for the balanced social hubs. In hypothesis 3, we predicted that larger clustering coefficients would lead to longer lifespan of social hubs. However, this hypothesis is rejected. Contrary to our expectation, clustering coefficients are negatively correlated with life$\operatorname{span}(\beta=-1.87, p<.01)$. We hypothesized that higher clustering coefficients would generate more dense local networks, and these dense networks would, in turn, nurture the local networks around the social hubs helping to sustain the social hub. However, it turns out that lower connectivity among the local neighbors of social hubs support longer lifespans for the social hubs.
The results imply that the key drivers of lifespan of social hubs are the norm of reciprocity and dominance. Social hubs have a stronger desire to increase self-esteem, gain respect from others and attain status in the social network. This high desire for self-esteem make these social hubs try harder to reciprocate visits they receive from their neighbors. This reciprocal behavior leads to longer lifespan of social hubs. We argued that the more dominating social hubs are in their local communities, the more active they become in collecting and disseminating information in order to maintain their social status. Increasing dominance power in a local community plays an important role in building and maintaining relationship with their neighborhoods, and as a result, it is associated with longer lifespan of social hubs. 


\section{Conclusions}

\subsection{Discussion}

Previous studies have shown that social hubs are of particular interest in social networks. In this paper, we have studied the social hubs further finding characteristics of the agents and factors that affect their lifespan as social hubs.

We find in agreement with prior work that younger agents are more prevalent in the hyperactive population than in the overall network (peak hyperactivity is in the 18-24 age range). We find a new result that indicates that people in their early 20s are much more sensitive to the norm of reciprocity while those in their late 20s are more motivated by dominance.

Our finding that social hubs have a short lifespan (average 2.5 months) is a potentially important observation. This indicates that in this virtual social network, the role of social hubs is not fixed to specific members. We observe that different members take the role of "social hubs" in various periods of time. Most of the social hubs have lifespans less than 3 months.

We find that the norm of reciprocity is very strong for social hubs. The social hubs are highly sensitive to paying back the visits by others. This may cause a ceiling effect to the social hubs since time and effort are a limited resource for them. Up to a certain level, social hubs are able to maintain the reciprocal behav- ior of visiting those who visit the social hubs.

We also find that dominance over the local neighbors in terms of activity is very strong for social hubs. Long-lived social hubs tend to have neighbors with low levels of activities. These results indicate that "social capital" in these young people's virtual social networks is of a clearly different structure than what has usually been found (communities of dense interaction without dominant individuals) for real-world communities. Indeed, this finding and the short lifespan of social hubs raises some questions about the sustaining value of hyperactive members on SNS. Their activity is presumably critical to overall activity and life in a SNS and this is how they have come to be viewed as critical to a healthy social network. However, their influence on lowering local social interaction while dominating the local network may well be non-sustaining.

\subsection{Managerial Implications}

Managers need to understand the behaviors of social hubs in terms of the norm of reciprocity and dominance in the local communities. In particular, it is important to understand what behaviors of an agent lead to the reciprocal behaviors of the partner agent, and which agent's behaviors achieve dominance over time. Once managers are able to identify those agents with high dominance, they need to tailor the incentive mechanism with the behaviors of 
agents with high dominance.

Managers, when they implement an incentive mechanism for agents to maintain their activities, need to pay attention to the age group they are targeting. Since late teen and early twenties agents are more sensitive to the norm of reciprocity, the incentive mechanism needs to focus on the reciprocal behaviors and the feedback behaviors. However, late twenties are more concerned with dominant status in their local communities, and as a result, they would be more sensitive to an incentive mechanism which favors the dominant status in member hierarchy.

Managers need to determine the overall value of social hubs in their SNS and develop appropriate strategies. This is difficult to do given the conflict between overall activity and local social coherence found in this work. Thus, the first priority should be to put emphasis on behaviors and variables not subject to such uncertainty. In particular, managers need to develop specific policies to encourage the norm of reciprocity among members as this encourages social hubs and other SNS members.

The norm of reciprocity is strengthened when there exists a positive feedback response from those with whom the social hubs are interacting (Resnick 2004). The positive feedback response from the local neighbors can be interpreted by social hubs and other agents as a sign of recognition by their peer group. Social hubs increase their involvement level based on the positive feedback from their peer group and in their reciprocal behaviors give encouraging feedback to other agents. Thus, a feedback mechanism plays an important role in influencing the norm of reciprocity for the social hubs in the social network (Dellarocas 2003).

In an example of a feedback mechanism, online game communities give players various ranks (e.g., VIP, general, sergeant, soldier, newbie) based on the length and level of their participation in the community. This kind of recognition motivates the most active members of the community to maintain and even deepen their participation in it, adding to the richness of the community as a whole. Another example is the "birthday" feature of Facebook, which prompt the members to send birthday greetings to friends (Ellison, Steinfield, and Lampe 2007). These features make the members more accessible to their friends and other members of Facebook, which in turn makes the user network more connected. The more connected network again improves the overall accessibility of its members. Thus, this creates a virtuous cycle between network connectivity and member accessibility.

A second motivational driver for social hubs is dominance in their local network but this implies being dominated for others and seems to result in less desirable reduction in local social interaction. Thus, managers need to more carefully consider how they treat dominant behavior. Dominance is strengthened when social hubs make connections with a large group 
of members and become more central figures among their peer groups. It has been found in many complex networks that there is a negative relationship between the dominance of a node and its clustering coefficient. More specifically, the clustering coefficient of an agent has been found to be inversely correlated with the number of connections of the agent (Ravasz and Barabási 2003). Burt (1997) argues that social hubs stand at the crossroads of a large social network and have the option of bringing together otherwise disconnected members in the network. Social hubs, because of their large numbers of connections within the network, play an important role in terms of information flow in the network. They know where all the "good" information is located. As long as they enjoy the trust of other members of the network, they are able to gather the "good" information from many people and distill it in ways that make it useful to others. However, the short lifespan of social hubs uncovered in this study makes this key connectivity function a temporary activity and whether this "dynamic turnover" is beneficial is not known. Overall, in designing and managing networks, encouraging dominance is a risky strategy but it is likely that giving members incentives to be connected to other members is very healthy for the SNS.

Facebook announced a new service called 'Facebook Connect' in order to create more opportunity for its members to interact with each other. Outside sites can become 'Facebook
Connect' partners, and become able to offer their visitors the option to log on Facebook and continue to interact with their Facebook friends even when they aren't at the Facebook site (IHT 2010).

A key issue for social network sites is how to increase the activity level of their core users. Facebook's decision to open its site to outside developers in 2007 was important for Facebook becoming the top website in terms of membership size and traffic volume. The large volume of applications and their download frequency shows significant activity from the members of Facebook and may support the goal of enhancing interaction with each other. We find that norm of reciprocity is very strong for social hubs. The social hubs are highly sensitive to paying back the visits made by others. This may cause a ceiling effect for the social hubs since time and effort are a limited resource for them. It is probably at least partly the cause of the dynamic turnover. Up to a certain level and for a given period, social hubs are able to maintain the reciprocal behavior of visiting those who visit the social hubs.

We also find that dominance in terms of $\mathrm{ac}^{-}$ tivity over the local neighbors is very strong for social hubs. Long-lived social hubs tend to have neighbors with low level of activities and low level of connections among them. These results indicate that social interactions in these young people's virtual social networks is of a clearly different structure than what has usually been 
found (communities of dense interaction without dominant individuals) for real-world communities. This is a dilemma for the managers of virtual social networks since the coherence of local network may work positively for the longevity of the social network, but it may have negative influence on the longevity of social hubs who are central to the social network.

\subsection{Limitations and Future Research}

Although the proportional hazard model and regression analysis identify important factors that impact the lifespan of social hubs, there is still much unexplained variation. Thus, it appears that a large part of the lifespan of social hubs may also depend on their own needs and personality factors. These are not able to be studied in an anonymous study such as constructed here but this is one valuable area for future research. Similarly, this study is limited because it is for only one SNS from one country with its own culture. It would be quite interesting to see if the influence of age, dominance and reciprocity was present in other SNS and other cultures.

Other more general future research topics are suggested by our work. A first such topic involves the key dilemma between the influences of social hubs on overall reciprocal activity in a network in opposition to dominant local behavior which may suppress local social interaction. Deeper research on users of SNS might be able to explicate this dilemma and identify more closely the motivations of a wider variety of users in terms of social interaction, dominance and reciprocity- the key behaviors shown in the current study. A second general area suggested by the current work is to investigate more generally the influence of time (dynamics) in social networks rather than just the structural aspects as often studied.

Finally, the driving force behind social hub's lifespan in our study is uncovering the relationship between explanatory variable and marketing performance, specifically marketing ROI or revenue in company. We hope that further study might reveal even deeper insight into social influence, network effects and marketing performance.

〈Received January 3. 2015〉

〈Revised February 21. 2015〉

〈Accepted March 23. 2015〉

\section{References}

Allison, P. D (1995), Survival analysis using SAS: A Practice Guide, SAS Institute Inc. Anagnostopoulos, Aris, Ravi Kumar, and Mohammad Mahdian (2008), "Influence and Correlation in Social Networks," Proceedings of the 14th ACM SIGKDD international conference on knowledge discovery and data mining (pp. 7-15). Las Vegas: ACM. 
Barabási, Albert-László and Reka Albert (1999), "Emergence of Scaling in random networks," Science, 286, 509-512. (2002). Linked: The New Science of Networks. Perseus Publishing.

, H. Jeong, Z. Neda, E. Ravasz, A. Schubert, and T. Vicsek (2002), "Evolution of the social network of scientific collaborations," Physica A, 311, 590-614. and Eric Bonabeau (2003), "Scale-Free Networks," Scientific American, 288(5), 50-59.

Bolton, Ruth N. (1998), “A Dynamic Model of the Duration of the Customer's relationship with a Continuous Service Provider: The Role of Satisfaction," Marketing Science, 17(1), 45-65.

Braha, Dan and Yaneer Bar-Yam (2006), "From Centrality to Temporary Fame: Dynamic Centrality in Complex Networks," Complexity, 12(2), 1-5.

Burt, Ronald S. (1997), "The Contingent Value of Social Capital," Administrative Science Quarterly, 42(2), 339-358.

Carr, Christopher L. (2006), "Reciprocity: The Golden Rule of IS-User Service Relationship Quality and Cooperation," Communications of the ACM, 49(6), 77-83.

Charla, Mathwick, Caroline Wiertz, and Ko de Ruyter (2008), "Social Capital Production in a Virtual P3 Community," Journal of Consumer Research, 34, 832-849.

CNN (2006), "Cyworld ready to attack
MySpace," http://money.cnn.com/2006/ 07/27/technology/cyworld0727.biz2/index. htm (accessed July 27, 2006)

Coleman, James (1990), Foundations of Social Theory. Harvard University Press.

Constant, David, Lee Sproull, and Sara Kiesler (1996), "The Kindness of Strangers: The Usefulness of Electronic Weak Ties for Technical Advice," Organizational Science, 7(2), 119-135.

Cox, D. R (1972), "Regression Modes and Life Tables," Journal of the royal Statistical Society, Series B(Methodological), 34, 187220.

Dellarocas, C. (2003). "The digitization of word of mouth: Promise and challenges of online feedback mechanisms," Management Science, 49(10), 1407-1424.

Dhanaraj, Charles and Arvind Parkhe (2006), "Orchestrating Innovation Networks," Academy of Management Review, 31(3), 659669.

Dorogovtsev, S. N. and J. F. F. Mendes (2002), "Evolution of Networks" Advances in Physics, 51(4), 1079-1187. (2003), Evolution of Networks: From Biological Nets to the Internet and WWW, Oxford University Press.

Doyle, John C., David L. Alderson, Steven Low, Matthew Roughan, Stanislav Shalunov, Reiko Tanaka, and Walter Willinger (2005), "The "Robust yet fragile" nature on the Internet," Proceedings of the National Aca- 
demy of Sciences of the United States of America, 102(41), 14497-14502.

Ellison, Nicole B., Charles Steinfield, Cliff Lampe (2007), "The Benefits of Facebook Friends: Social Capital and College Students' Use of Online Social Network Sites," Journal of Computer-Mediated Communications, 12(4), Article 1, http://jcmc.indiana.edu/vol12/ issue4/ellison.html, (accessed July 15, 2007).

Farewell, V. T. and R. L. Prentice (1980), "The approximation of partial likelihood with emphasis on case-control studies," Biometrika, 67(2), 273-278.

Gladwell, Malcolm. (2000), The Tipping Point. New York, Little, Brown and Company. Giesler, Markus (2006), "Consumer Gift Systems," Journal of Consumer Research, 36(4), 283290.

Goldenberg, Jacob, Sangman Han, Donald R. Lehmann, and Jae Won Hong (2009), “The Role of Hubs in the Adoption Process" Journal of Marketing, 73(2), 1-13.

Iacobucci, Dawn (1998), "Interactive Marketing and the Meganet: Networks of Networks," Journal of Interactive Marketing, 12(1), 5-16. Iyengar, Raghuram, Christophe Van den Bulte, and Thomas W. Valente (2010), "Opinion Leadership and Social Contagion in New Product Diffusion," Marketing Science, Forthcoming.

IHT (2010), Attraction of numbers adds value to Facebook. March 8, 2010.

Katona, Zsolt, Zubcsek, Peter P., and Miklos
Sarvary (2011), "Network Effects and Personal Influences: The Diffusion of an Online Social Network," Journal of Marketing Research, 48(3), 425-443.

Kavanaugh, Andrea. And Scott J. Patterson (2001), "The impact of Community Computer Networks on Social Capital and Community Involvement," American Behavioral Scientist, 45(3), 496-509.

Kleinberg, Jon M. (1999), “Authoritative Sources in a Hyperlinked Environment," Journal of the ACM, 46(5), 604-632.

Kratzer, Jan and Christopher Lettl (2009), "Distinctive Roles of Lead Users and Opinion Leaders in the Social Networks of Schoolchildren," Journal of Consumer Research, 36, 646-659 (December).

Leary, Mark R., Catherine A. Cottrell, and Misha Phillips (2001), "Deconfounding the effects of dominance and social acceptance on self esteem," Journal of Personality and Social Psychology, 81(5), 898-909.

Mikami, Shunji (2002), "Internet Use and Sociability in Japan," IT \& Society, 1(1), 242250.

Newman, Mark E. (2003), “The structure and function of complex networks," SIAM Review, 45, 167-256.

(2005), "Power laws, Pareto distributions and Zipf's law," Comtemporary Physics, 46(5), 323-351.

Oh, Wonseok and Sangyong Jeon (2007), "Membership Herding and Network Stability in 
the Open Source Community: The Ising Perspective," Management Science, 53(7), 1086-1101.

Onyx, Jenny and Paul Bullen (2000), "Measuring Social Capital in Five Communities," Journal of Applied Behavioral Science, 36(1), 23-42.

Price, D. J. de Solla (1965), "Networks of Scientific Papers” Science, 149, 510-515. . (1976), “A general Theory of Bibliometric and other Cumulative Advantage Processes," Journal of the American Society of Information Science, 27(5-6), 292-306.

Putnam, Robert D. (1995), “Turning in, turning out: The strange disappearance of social capital in America," PS: Political Science \& Politics, 28(4), 664-683.

Ravasz, Erzsébet and Albert-László Barabási (2003), "Hierarchical organization in complex networks," Physics review E, 67(2), 026112. Reinartz, Werner J. and V. Kumar (2003), “The Impact of Customer Relationship Characteristics on Profitable Lifetime Duration," Journal of Marketing, 67(1), 77-99.

Resnick, M. (2004), “New Styles of Thinking for the Era of the Organic Network," BT Technology Journal, 22(4), 113-120.

Stephen, Andrew T and Olivier Toubia (2010), "Deriving Value from Social Commerce Networks," Journal of Marketing Research, 47(2), 215-228.

The New York Times (2010), "World's Largest Social Network: The Open Web," http:// www.nytimes.com/2010/05/16/business/16 digi.html, (accessed May 14, 2010).

Thorn, Brian K. and Terry Connolly (1987), "Discretionary Data Bases: A Theory and Some Experimental Findings," Communication Research, 14(5), 512-528.

Watts, Duncan J. (2004), "The new science of networks," Annual Review of Sociology, 30, 243-270. and Steven H. Strogatz (1998), “Collective dynamics of 'small-world' networks, Nature, 393, 440-442.

Wellman, Barry and Milena Gulia, (1999), Net Surfers Don't Ride Alone: Virtual Communities as Communities. Networks in the Global Village, edited by Barry Wellman, Boulder, CO, Westview Press. 